\title{
The software in the middle_-Letter from the Editors-in-Chief
}

\author{
Gordon Blair · Fabio Kon
}

Published online: 24 August 2010

(C) The Brazilian Computer Society 2010

Welcome to the second issue of JISA. As Editors-in-Chief, we have been delighted by the response to the inaugural issue that included some quite outstanding papers from major figures in the field on Internet Services and Applications. We aim to maintain this high standard and, through this, get JISA quickly established as one of the major publication venues for work in this field. To achieve this goal, we need your help and encourage you to submit high quality manuscripts to us for consideration. In return, we promise fast turnaround in terms of reviewing times.

This second issue features four papers, including three papers that together form a special issue on the topic of middleware.

The first paper, "A Search Engine for the Global PKI" by Paul Rabinovich, focuses on the problem of managing Public Key Infrastructures. The author seeks to offer a service for global PKI matching, what Google does for the Web, offering a unified view of the global PKI and supporting such functions as certificate discovery and certification revocation status lookup. These are important issues in being able to offer practical solutions for PKI management in large-scale distributed systems. Congratulations to Paul on being our first accepted author through the open submission process (the first issue was devoted to invited papers).

The next three papers form a special issue on the topic of middleware. For this, we have formed an alliance with the ACM/IFIP/USENIX Middleware Confer-

\section{G. Blair}

Computing Department, Lancaster University, Lancaster, UK

e-mail: gordon@comp.lancs.ac.uk

F. Kon $(\bowtie)$

Department of Computer Science, Institute of Mathematics and

Statistics, University of São Paulo, São Paulo, Brazil

e-mail: fabio.kon@ime.usp.br ence (http://www.middleware-conference.org), which recently celebrated its tenth anniversary. In particular, we have selected research works that received the best paper awards in 2007, 2008, and 2009 and requested authors to produce extended and updated versions for this special issue. Middleware is an important topic for Internet Services and Applications, especially given the increasing complexity and heterogeneity of modern networked environments. Their role is to simplify the programming of associated services and applications and, where possible, mask out such complexities.

The initial paper in this theme is "Unifying Divergence Bounding and Locality Awareness in Replicated Systems with Vector-Field Consistency" by Veiga et al. This paper looks at replication algorithms for applications such as multiplayer games that take advantage of context information (particularly the position of players) to optimize performance and also adapt the consistency-enforcement approach based on current game state. These two approaches are unified within an overall framework based on the concept of Vector-Field Consistency. Overall, this paper shows the advantages of adaptive approaches to demanding application areas such as multiplayer games.

The second paper, by Ningfang Mi et al., is called "Sizing Multi-Tier Systems with Temporal Dependence: Benchmarks and Analytic Models". This paper examines the effects of burstiness in multi-tier systems, a heavily deployed approach in contemporary distributed systems. The authors argue that existing models cannot predict the performance of such bursty environments and hence propose a methodology, based on an index of dispersion, to integrate workload burstiness into models and benchmark tools. Validation of the approach is also offered based on measurements on real testbeds. 
The final paper is on the "Dynamic Deployment and Reconfiguration of Ad Hoc Routing Protocols" by Rajiv Ramdhany et al. This paper deals with the high level of heterogeneity in the field of routing protocols for mobile ad hoc networks (MANETs) where large numbers of protocols have been proposed. In particular, the paper describes a framework that supports the deployment of customized MANET protocols and also the co-existence of multiple protocol instances allowing the system to be adapted at run-time to the current operational conditions. The framework also supports the development of new or hybrid protocols based on the features of others. The underlying framework adopts a component-based approach and demonstrates the capability of such approaches in supporting more configurable and reconfigurable protocols.

We hope you enjoy this second issue of JISA and also the insight into current research topics in the field of middleware.

August, 2010

Gordon Blair and Fabio Kon

Editors-in-Chief 